\title{
Enfermedad de Camurati-Engelmann
}

\author{
Camurati-Engelmann disease
}

\author{
Lina Johanna Moreno-Giraldo,* Stephany Ramírez-Ramírez, ${ }^{\ddagger}$ \\ Daniela Arturo-Terranova, ${ }^{\ddagger}$ José María Satizábal-Soto ${ }^{\S,+}$ \\ * Universidad Santiago de Cali, Universidad Libre, Postgrado en Ciencias Biomédicas-Genética \\ Médica Universidad del Valle; ${ }^{\ddagger}$ Universidad del Valle, Cali; ${ }^{\S}$ Énfasis Genética Médica, Universidad \\ Santiago de Cali, Postgrado en Ciencias Biomédicas. Genética Médica Universidad del Valle. \\ Grupo de Investigación Enfermedades Congénitas del Metabolismo, Cali, Colombia.
}

\begin{abstract}
RESUMEN
Introducción: La enfermedad de Camurati-Engelmann es una displasia ósea esclerosante autosómica dominante, se estima en 1:1,000,000 de personas, con aproximadamente 300 casos reportados en el mundo. Las mutaciones en el gen TGFB1 se han asociado con el desarrollo de la enfermedad. El objetivo es describir clínica y molecularmente a una lactante colombiana con la enfermedad. Caso clínico: Paciente femenino de 22 meses, producto del primer embarazo de padres no consanguíneos sin historia familiar de enfermedades, con cuadro clínico de debilidad en extremidades, signos de compromiso de sistema osteo-artro-neuromuscular, sospechando displasia ósea. Se realizó estudio molecular mediante secuenciación de exoma completo y estudio genómicobioinformático utilizando tecnología in-silico, encontrando una mutación missense (c.466C>T;p.Arg156Cys) en el gen TGFB1; por bioinformática se determinó que esta variante genética tenía significado clínico patogénico. Conclusión: Este caso proporciona datos actualizados de la enfermedad de Camurati-Engelmann, resaltando la importancia de las técnicas genómicas y bioinformáticas para el diagnóstico.

Palabras clave: Camurati-Engelmann, contractura, lactante, malformación osteo-artro-neuromuscular, secuenciación exoma.
\end{abstract}

\begin{abstract}
Introduction: Camurati-Engelmann disease is an autosomal dominant sclerosing bone dysplasia, estimated at 1:1,000,000 people, with approximately 300 cases reported worldwide. Mutations in TGFB1 gene have been associated with the development of the disease. The objective is to describe clinically and molecularly a Colombian infant with this disease. Clinical case: 22-month-old female patient, daughter of the first pregnancy of non-consanguineous parents, with no family history of diseases. The clinical picture consisted of limb weakness, signs of compromise of the osteo-arthroneuromuscular system, hence, bone dysplasia was suspected. Molecular study by complete exome sequencing and genomic-bioinformatics study, using in-silico technology, was performed. A missense mutation (c.466C> T; p.Arg156Cys) was found in the TGFB1 gene; by bioinformatics, it was determined that this genetic variant had pathogenic clinical significance. Conclusion: This case provides data from the Camurati-Engelmann disease, highlighting the importance of genomic and bioinformatics techniques for its diagnosis.
\end{abstract}

Keywords: Camurati-Engelmann, contracture, infant, osteoarthro-neuromuscular malformation, exome sequencing.

\footnotetext{
+Correspondencia: JMSS, jose.satizabal@correounivalle. edu.com

Conflicto de intereses: Los autores declaran que no tienen. Citar como: Moreno-Giraldo LJ, Ramírez-Ramírez S, ArturoTerranova D, Satizábal-Soto JM. Enfermedad de Camurati-Engelmann. Rev Mex Pediatr. 2020; 87(6); 221-226. https://dx.doi. org/10.35366/97684
}

\section{INTRODUCCIÓN}

La enfermedad de Camurati-Engelmann (ECE) (MIM 31300, ORPHA: 1328, CIE-10: Q783) está relacionada con mutaciones en el factor de crecimiento transformante $\beta 1$ (TGFB1), el cual participa en la proliferación ósea. La mayoría (82.2\%) de las mutaciones ocurren en el exón 4, que codifica la región en el péptido asociado a 
la latencia $T G F B 1$ que rodea los residuos responsables de la homodimerización (Cys223 y Cys225). ${ }^{1}$ El segundo exón más afectado es el exón 1 y los tres alelos mutantes más frecuentes son: p.Arg210Cys, p.Arg218His, y p.Cys225Arg. ${ }^{2}$

Bajo condiciones fisiológicas TGFB1 estimula la formación de hueso y suprime el hueso por reabsorción. Por lo tanto, la interrupción de la función de los osteoblastos y los osteoclastos conduce a la hiperostosis a lo largo de la diáfisis de los huesos largos y la esclerosis del esqueleto, produciendo los síntomas característicos de la enfermedad. ${ }^{1-3}$

La frecuencia de la ECE se estima en un caso por millón de personas; hasta el momento se calcula que hay 300 casos reportados en todo el mundo. ${ }^{1,3} \mathrm{El}$ inicio de la enfermedad es en la niñez, progresando durante la adolescencia, pero en la edad adulta su curso puede ser estacionario o lentamente progresivo. Los huesos afectados en orden de frecuencia son: fémur, tibia, peroné, húmero, cúbito y radio. ${ }^{3} \mathrm{Es}$ posible realizar un diagnóstico prenatal cuando se ha identificado la mutación causante de la enfermedad en la familia. ${ }^{4}$

Las principales características clínicas de la ECE se reportan en la Tabla 1, en la que se compara el caso presentado con otros reportados en la literatura. En una revisión de 100 casos de la enfermedad realizada por Janssens y cols., en 2006, se evidenció que $94 \%$ de los pacientes con el defecto molecular en TGFB1 presentó síntomas radiológicos, siendo el engrosamiento cortical de las diáfisis de los huesos largos la primera manifestación. El cráneo y la pelvis fueron otros sitios involucrados. Asimismo, describen que $74 \%$ es sintomático, $55 \%$ con síntomas de gravedad leve a moderada. Los síntomas más comunes fueron dolor en las extremidades en $68 \%$, un modo peculiar de caminar en $48 \%$, fatiga fácil en $44 \%$ y debilidad muscular en $39 \%$. La reducción de la grasa subcutánea se presentó en $21 \%$ y la pérdida auditiva en $15 \%$. En la revisión se describen las diferentes variantes genéticas: $28 \_36$ dup y $241 \mathrm{~T}>\mathrm{C}$ en el exón $1 ; 463 \mathrm{C}>\mathrm{T}$ en el exón $2 ; 653 \mathrm{G}>\mathrm{A}, 652 \mathrm{C}>\mathrm{T}, 664 \mathrm{C}>\mathrm{G}, 667 \mathrm{~T}>\mathrm{C}, 667 \mathrm{~T}$ $>$ G y $673 \mathrm{~T}>\mathrm{C}$ en el exón $4 .^{5}$

Collet y cols. reportaron la variante c. $466 \mathrm{C}>\mathrm{T}$, p.Arg 156 Cys, en Francia, en una familia de 10 integrantes afectados por una forma atípica de la ECE, caracterizada por signos radiológicos leves limitados a la tibia y asociados con sobrepeso u obesidad. Los pacientes presentaron hiperostosis tibial diafisaria aislada, conocida también como enfermedad de Ribbing y una edad de inicio tardía, (personas mayores de 30 años). Esta mutación se había informado previamente en una familia marroquí,,${ }^{6,7}$ con un fenotipo similar, lo que sugiere una posible relación fenotipo/ genotipo.

En 2019, Hughes y cols. analizaron 10 casos de una familia de Nueva Zelanda (la mitad eran asintomáticos), encontrando la mutación $\mathrm{R} 218 \mathrm{H}$ en el gen TGFB $1 .{ }^{8}$ En Colombia, Vega-Caicedo y su equipo reportaron un paciente de 11 años con hallazgos radiológicos y clínicos sugestivos de la enfermedad, con una fractura patológica subtrocantérica como complicación. ${ }^{9}$ También, Restrepo y cols. presentaron el caso de una mujer de 54 años con ECE, quien solamente tenía compromiso tibial, sin otra sintomatología; ${ }^{4} \sin$ embargo, en estos dos estudios no se describen las variantes genéticas.

La bioinformática es una disciplina que tiene como objetivo la aplicación de tecnologías computacionales y estadísticas para la gestión de datos biológicos. Ha permitido comprender los fundamentos de las enfermedades, especialmente en el campo de la genética, constituyéndose en la principal herramienta de análisis de datos masivos que puede ser aplicada en la clínica. ${ }^{10}$ Una de las tecnologías más utilizadas en la actualidad es la secuenciación de próxima generación (NGS). Por medio de esta técnica contribuyen a la consejería genética.

El tratamiento para la ECE consiste en el empleo de glucocorticoides que limitan el aumento de la formación ósea, al disminuir la masa ósea mediante la apoptosis de osteoblastos, proliferación de osteoclastos y disminución de la absorción de calcio. Otros medicamentos son bifosfonatos y antiinflamatorios no esteroideos. ${ }^{11}$

Los diagnósticos diferenciales de la ECE son displasia craneodiafisaria, síndrome de Kenny-Caffey, enfermedad de Paget juvenil, displasia hematodiafisaria de Ghosal, osteoesclerosis autosómica dominante de tipo Worth, esclerosteosis y la hiperostosis cortical.

Este estudio tiene como objetivo describir clínica y molecularmente una lactante colombiana con ECE; buscando contribuir al reconocimiento temprano, entendimiento y diagnóstico de esta condición.

\section{PRESENTACIÓN DEL CASO}

Lactante femenino de 22 meses, producto del primer embarazo de padres no consanguíneos sin historia familiar de enfermedades. Nació por parto vaginal, a las 32 semanas de gestación, con requerimiento 
Tabla 1: Síntomas clínicos de la enfermedad de Camurati-Engelmann del caso estudiado y reportados en la literatura.

\begin{tabular}{|c|c|c|c|c|c|c|c|c|c|c|c|c|c|}
\hline \multicolumn{14}{|c|}{ Síntomas clínicos } \\
\hline Estudio & $\begin{array}{l}\text { Variante } \\
\text { ADN }\end{array}$ & $\begin{array}{l}\text { Variante } \\
\text { proteica }\end{array}$ & $\mathrm{DE}$ & FF & DM & MP & PA & GR & PV & DC & I & $\mathbf{S}$ & Otros \\
\hline Caso actual 2020 & $466 C>T$ & $\mathrm{~A} 156 \mathrm{C}$ & $x$ & & $x$ & & & $x$ & & & & & \\
\hline $\begin{array}{l}\text { Simsek S et al, } 2005 \\
\text { Janssens et al, } 2006\end{array}$ & $466 \mathrm{C}>\mathrm{T}$ & $\mathrm{A} 156 \mathrm{C}$ & $x$ & & $x$ & & & & & & & $x$ & Osteomielitis crónica \\
\hline Familia Bélgica & $673 T>C$ & C225R & $x$ & & & $x$ & & $x$ & & & & & Hipertermia \\
\hline Familia Iraq & $653 G>A$ & $\mathrm{R} 218 \mathrm{H}$ & $x$ & $x$ & $x$ & $x$ & $x$ & $x$ & & $x$ & & & $\begin{array}{l}\text { Hepatoesplenomegalia, estatura } \\
\text { pequeña, compresión de nervio } \\
\text { craneal }\end{array}$ \\
\hline Familia Reino Unido & $652 \mathrm{C}>\mathrm{T}$ & $\mathrm{R} 218 \mathrm{C}$ & $x$ & $x$ & $x$ & $x$ & & & & & & & ESR y CRP aumentada \\
\hline Familia Italia & 28_36dup & L10-L12dup & & & & & $x$ & $x$ & & & & & \\
\hline Familia Bélgica & $241 T>C$ & Y81H & $x$ & $x$ & $x$ & $x$ & & $x$ & & & & & Estatura pequeña \\
\hline Familia Bélgica & $241 \mathrm{~T}>\mathrm{C}$ & Y81H & $x$ & $x$ & $x$ & $x$ & & & & & & & \\
\hline Familia Italia & $653 \mathrm{G}>\mathrm{A}$ & $\mathrm{R} 218 \mathrm{H}$ & $x$ & $x$ & $x$ & $x$ & & $x$ & & & & & \\
\hline Familia Alemania & $653 \mathrm{G}>\mathrm{A}$ & $\mathrm{R} 218 \mathrm{H}$ & $x$ & $x$ & $x$ & $x$ & & & & & & & \\
\hline Familia Reino Unido & $653 G>A$ & $\mathrm{R} 218 \mathrm{H}$ & $x$ & $x$ & & & & & & & & & \\
\hline Familia Oceanía & $653 G>A$ & $\mathrm{R} 218 \mathrm{H}$ & & & & & $x$ & & & & & & Proptosis \\
\hline Familia Marruecos & $463 \mathrm{C}>\mathrm{T}$ & $\mathrm{R} 156 \mathrm{C}$ & $x$ & $x$ & & $x$ & & & & & & $x$ & ESR y CRP aumentada \\
\hline Familia Bélgica & $673 T>C$ & C225R & $x$ & $x$ & $x$ & $x$ & & & & & & $x$ & \\
\hline Familia España & $652 \mathrm{C}>\mathrm{T}$ & $\mathrm{R} 218 \mathrm{C}$ & $x$ & & $x$ & $x$ & & & $x$ & & & & $\begin{array}{l}\text { Esplenomegalia leve, parálisis } \\
\text { facial, hipertensión }\end{array}$ \\
\hline Familia Alemania & $664 C>G$ & H222D & $x$ & $x$ & $x$ & $x$ & & $x$ & & & & & Pubertad tardía, estatura baja \\
\hline Familia Israel & $652 \mathrm{C}>\mathrm{T}$ & $\mathrm{R} 218 \mathrm{C}$ & $x$ & NR & $x$ & $x$ & & NR & & & & & $\begin{array}{l}\text { Inhabilidad para correr rápida- } \\
\text { mente }\end{array}$ \\
\hline Familia Japón & $673 T>C$ & C225R & $x$ & NR & $x$ & $x$ & $x$ & NR & & & & & $\begin{array}{l}\text { Hábito marfanoide, parálisis del } \\
\text { nervio facial, pubertad tardía }\end{array}$ \\
\hline Familia Portugal & $653 G>A$ & $\mathrm{R} 218 \mathrm{H}$ & $x$ & $x$ & $x$ & $x$ & NR & & & $x$ & & & Apetito escaso, pubertad tardía \\
\hline Familia Francia & $652 \mathrm{C}>\mathrm{T}$ & $\mathrm{R} 218 \mathrm{C}$ & $x$ & $x$ & $x$ & $x$ & NR & NR & & & & & Apetito escaso \\
\hline Familia Bélgica & $673 T>C$ & C225R & $x$ & $x$ & $x$ & $x$ & NR & NR & & & & & \\
\hline Familia 20 Francia & $673 \mathrm{~T}>\mathrm{C}$ & C225R & $x$ & $x$ & $x$ & $x$ & NR & NR & & $x$ & & & Apetito escaso \\
\hline Familia Australia & $673 T>C$ & C225R & $x$ & $x$ & $x$ & $x$ & NR & NR & & $x$ & & & Apetito escaso \\
\hline Familia Francia & $652 \mathrm{C}>\mathrm{T}$ & $\mathrm{R} 218 \mathrm{C}$ & $x$ & $x$ & $x$ & $x$ & NR & NR & & & & & Apetito escaso \\
\hline Familia Francia & $652 \mathrm{C}>\mathrm{T}$ & $\mathrm{R} 218 \mathrm{C}$ & $x$ & $x$ & $x$ & $x$ & NR & NR & & $x$ & & & Apetito escaso \\
\hline Familia EUA & $653 G>A$ & $\mathrm{R} 218 \mathrm{H}$ & $x$ & $x$ & $x$ & $x$ & $x$ & $x$ & & & & & Vértigo, pubertad tardía \\
\hline Collet C et al, 2013 & $466 C>T$ & $\mathrm{~A} 156 \mathrm{C}$ & $x$ & & & & & & & & & $x$ & $\begin{array}{l}\text { Esclerosis, diáfisis de huesos } \\
\text { largos }\end{array}$ \\
\hline Vega RA et al, 2013 & No reporta & No reporta & $x$ & & & & & & & & & & Diáfisis de huesos largos \\
\hline Restrepo JP et al, 2016 & No reporta & No reporta & $x$ & & $x$ & & & & & & $x$ & & \\
\hline Ge DH et al, 2018 & No reporta & No reporta & $x$ & & $x$ & & & & & & & & $\begin{array}{l}\text { Esclerosis, artrosis, diáfisis de } \\
\text { huesos largos }\end{array}$ \\
\hline Hughes P et al, 2019 & $653 \mathrm{G}>\mathrm{A}$ & $\mathrm{R} 218 \mathrm{H}$ & $x$ & & $x$ & $x$ & $x$ & & $x$ & $x$ & & & $\begin{array}{l}\text { Bajo peso, parálisis del nervio } \\
\text { facial }\end{array}$ \\
\hline Abdulla MC et al, 2019 & $652 \mathrm{C}>\mathrm{T}$ & $\mathrm{R} 218 \mathrm{C}$ & $x$ & $x$ & & & & & & & & & $\begin{array}{l}\text { Hábito marfanoide, diáfisis de } \\
\text { huesos largos }\end{array}$ \\
\hline
\end{tabular}

$\mathrm{NR}=$ no reporta, $\mathrm{DE}=$ dolor en extremidades, $\mathrm{FF}$ = fatiga fácil, $\mathrm{DM}=$ debilidad muscular, $\mathrm{MP}=$ marcha de pato, $\mathrm{PA}=$ pérdida auditiva, $\mathrm{GR}=$ grasa subcutánea reducida, $\mathrm{PV}=$ pérdida de visión, $\mathrm{DC}=$ dolor de cabeza, $\mathrm{I}=$ insomnio, $\mathrm{S}=$ sobrepeso, $\mathrm{ESR}=$ velocidad de sedimentación globular, $\mathrm{CRP}=$ proteína $\mathrm{C}$ reactiva. 
de oxígeno por mascarilla y con múltiples hospitalizaciones por traqueítis. Al mes de vida se le realiza gastrostomía y a los dos meses traqueostomía por disposición de distractores faciales, lo cual estaba asociado a debilidad en extremidades, contractura muscular, dificultad y alteración en la marcha, coxa valga, genu valgo y pies planos; por lo que se sospechó de displasia ósea. Por ecocardiograma, no se detectó cardiopatía congénita. Mientras que radiológicamente se confirma deformidad en pie equino varo bilateral y luxación congénita de cadera izquierda (Figura 1).

Se realizó secuenciación de exoma completo, utilizando la tecnología Illumina a partir de sangre venosa, todas las regiones seleccionadas tienen una profundidad mayor o igual a $32.2 \mathrm{x}$ y un umbral de confianza mínimo de mapeo de Q30 con una lectura total de 27,320,632 bibliotecas Nexteratm Illumina. Las lecturas se alinearon con la secuencia de referencia (GRCh37) y se realizó llamado de variantes.

Análisis in silico: para analizar las variantes reportadas se utilizaron diferentes tipos de software: Mutation Taster (http://www.mutationtaster.org/), PROVEAN (http://provean.jcvi.org/index.php), el predictor UMD (http://umd-predictor.eu/), POLYPHEN (http://genetics.bwh.harvard.edu/pph2/) y Clinvar (https://www.ncbi.nlm.nih.gov/clinvar/).

\section{RESULTADOS}

El análisis de secuencia del gen del factor de crecimiento transformante $\beta 1$ (TGFB1) reveló una mutación missense (c.466C>T; p.Arg156Cys), que consiste en una transición de citosina a timina en la posición 466, la cual modifica en la proteína el aminoácido, arginina por cisteína en la posición 156. Con los software de predicción, se estableció un significado clínico patogénico de la variante identificada (Figura 2).

\section{DISCUSIÓN}

El gen TFGB1 se localiza en el brazo largo del cromosoma 19 (19q13.2), codifica la proteína TFGB1, la cual desencadena señales que regulan diversas actividades celulares, incluyendo el crecimiento y proliferación, diferenciación, motilidad y la apoptosis celular. Se encuentra en todo el organismo, pero es particularmente abundante en el esqueleto, donde ayuda a la formación, crecimiento de hueso y cartílago. También está involucrada en la formación de vasos sanguíneos, desarrollo
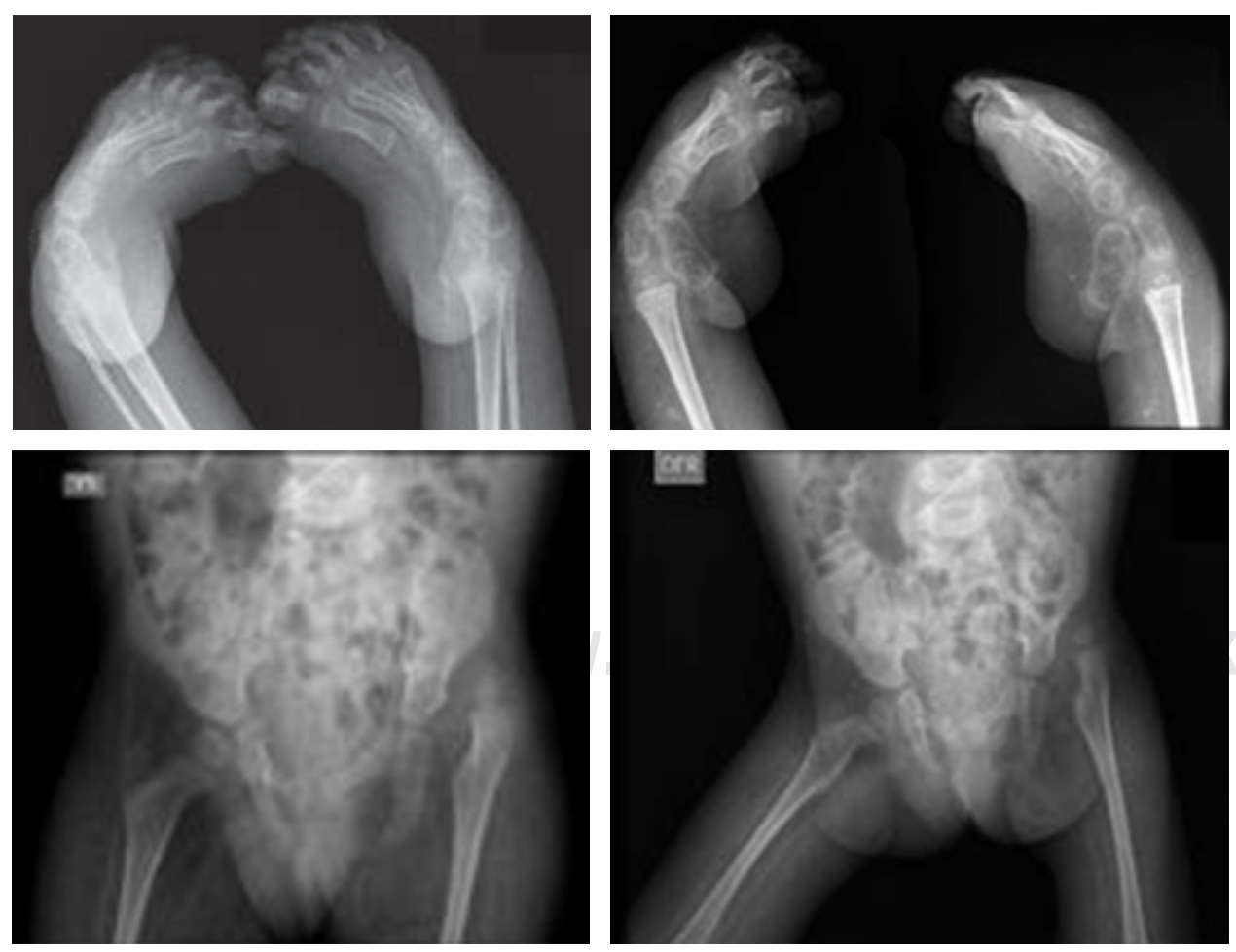

Figura 1:

Informe radiológico que confirma deformidad en pie equino varo bilateral y luxación congénita de cadera izquierda. 


\section{Proteína Acc Posición $\mathrm{AA}_{1} \quad \mathrm{AA}_{2}$ Descripción \\ Canónica; RecNombre: Completo = Transformador del factor de \\ P01137 156 R C crecimiento beta-1; Corto = TGF-beta- 1 ; Contiene: RecNombre: Completo = Péptido asociado a latencia; Corto = LAP; Banderas: Precursor; Largo: 390}

Resultados

Predicción-confianza

HumDiv

Se predice que esta mutación es probablemente dañina con un puntaje de 1.000 (sensibilidad: 0.00; especificidad: 1.00)

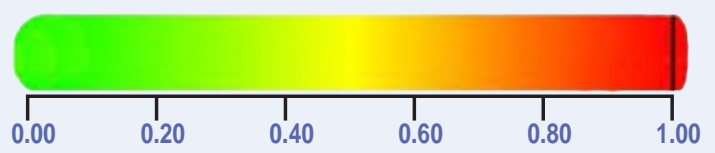

Figura 2:

Identificación de significado patogénico de la variante p.Arg156Cys con el programa bioinformático Polyphen. de tejido muscular, grasa corporal, la cicatrización de heridas, los procesos inflamatorios y la prevención de crecimiento tumoral. Las mutaciones en el gen TFGB1 dan lugar a una ganancia de función de TGFB1. Esta actividad anormal en la proteína provoca un aumento en la transducción de la señal, lo que da lugar a una mayor formación ósea.

Anteriormente, el diagnóstico del síndrome de ECE se basaba principalmente en sus manifestaciones clínicas y en las alteraciones radiográficas. ${ }^{12}$ Sin embargo, se requiere la confirmación molecular. ${ }^{13}$

Se ha descrito que los individuos gravemente afectados muestran un fenotipo caracterizado por macrocráneo con frente prominente, exoftalmia, extremidades delgadas con huesos gruesos y masa muscular escasa. Suelen presentar un aspecto grácil, hábito marfanoide, marcha anadeante con ampliación de la base de sustentación, disminución de la fuerza con reflejos osteotendinosos exaltados, piernas arqueadas, pies planos y valgos, lordosis lumbar y escoliosis. ${ }^{2,3}$

En los sujetos con ECE es importante la prevención de complicaciones secundarias, por lo tanto, su detección temprana es fundamental. En estudios a largo plazo se han observado anemia, hepatoesplenomegalia, compresión de pares craneales. ${ }^{2}$

La mutación missense (c.466C $>\mathrm{T} ;$ p.Arg156Cys) encontrada en la paciente del presente reporte, es la misma mutación del estudio de Collet y cols. y en la familia marroquí, mencionados anteriormente. Ésta es una forma atípica de la ECE, se caracteriza por signos radiológicos leves limitados a la tibia (Tabla 1).

\section{Consideraciones éticas}

Para la publicación de este caso, se obtuvo consentimiento informado por parte de los padres de la paciente.

\section{REFERENCIAS}

1. Ge DH, Yu S, Ziegler JD, Schwarzkopf R. Total hip arthroplasty in a patient with Camirati-Engelmann disease a case report. JBJS Case Connect. 2018; 8: 2. doi: 10.2106/jbjs.cc.17.00286.

2. García MD, Vargas C. Síndrome de Camurati-Engelmann. Sem Fund Esp Reumatol. 2011; 12(3): 91-97. https://doi.org/10.1016/j. semreu.2011.06.002.

3. Restrepo JP, Molina M. Enfermedad de Camurati-Engelmann: reporte de un caso y revisión de la literatura. Rev Colomb Reumatol. 2016; 23(3): 218-222. https://doi.org/10.1016/j. rcreu.2016.03.002.

4. Wallace SE, Wilcox WR. Camurati-Engelmann disease. GeneReviews, University of Washington, Settle. 2017. https:// www.ncbi.nlm.nih.gov/books/NBK1156/.

5. Janssens K, Vanhoenacker F, Bonduelle M, Verbruggen L, Van Maldergem L, Ralston $S$ et al. Camurati-Engelmann disease: review of the clinical radiological, and moleculardata of 24 families and implications for diagnosis and treatment. J Med Genet. 2006; 43: 1-11.

6. Simsek S, Janssens K, Kwee ML, Van Hul W, Veenstra J, Netelenbos JC. Camurati-Engelmann disease (progressive diaphyseal dysplasia) in a Moroccan family. Osteoporos Int. 2005; 16: 1167-1170. https://doi.org/10.1007/s00198-005-1896-2.

7. Collet C, Laplanche JL, de-Vernejoul MC. Camurati-Engelmann disease with obesity in a newly identified family carrying a missense p.Arg156Cys mutation in the TGFB1 gene. Am J Med Genet A. 2013; 161(8): 2074-2077. https://doi.org/10.1002/ ajmg.a.36022.

8. Hughes P, Hassan I, Que L, Mead P, Lee JH, Love DR et al. Observations on the Natural History of Camurati-Engelmann disease. J Bone Miner Res. 2019; 34: 875-882. https:// doi:10.1002/jbmr.3670. 
9. Vega RA, Muñoz JF, Amador JA, Lazala O. Enfermedad de Camurati-Engelmann: reporte de caso. Rev Col Or Tra. 2013; 27(2): 131-136. https://doi.org/10.1016/s0120-8845(13)70007-5.

10. Suarez-Obando F. La atención clínica de las enfermedades raras: Un reto para la educación médica. Medicina. 2018; 40: 228-241.

11. Abdulla MC. Camurati-Engelmann disease with good treatment response to Losartan. Indian J Nucl Med. 2019; 34: 223-225. https://doi:10.4103/ijnm.IJNM_44_19.
12. Rodríguez-Santiago B, Armengol L. Tecnologías de secuenciación de nueva generación en diagnóstico genético pre- y postnatal. Diag Prenat. 2012; 23(2): 56-66. https://doi.org/10.1016/j. diapre.2012.02.001

13. Ministerio de Salud y Protección Social. Resolución número 005265 de 2018. Listado de enfermedades huérfanas. Disponible en: https://www.minsalud.gov.co/sites/rid/Lists/BibliotecaDigital/ RIDE/DE/DIJ/resolucion-5265-de-2018.pdf. 